\title{
Temperature tolerance analysis on the volume holographic data storage (Withdrawal Notice)
}

Liangcai Cao, Shenghan Wu, Song Zong, Guofan Jin

Liangcai Cao, Shenghan Wu, Song Zong, Guofan Jin, "Temperature tolerance analysis on the volume holographic data storage (Withdrawal Notice)," Proc. SPIE 10384, Optical Data Storage 2017: From New Materials to New Systems, 1038409 (17 January 2018); doi: 10.1117/12.2274910

SPIE Event: SPIE Optical Engineering + Applications, 2017, San Diego, California, United States 


\section{Temperature tolerance analysis on the volume holographic data storage (withdrawal notice)}

Liangcai Cao, Shenghan Wu, Song Zong, Guofan Jin

Tsinghua Univ. (China)

Proc. SPIE 10384, 1038409 (2017)

Withdrawn from Publication: 15 January 2018

Conference Date: 6 August 2017

Conference Location: San Dieog, California, United States

Conference Title: Optical Data Storage 2017: From New Materials to New Systems

Conference Chairs: Ryuichi Katayama, Yuzuru Takashima

Publisher's Note: The conference presentation was withdrawn per author request. 